\title{
NEK2 serves as a prognostic biomarker for hepatocellular carcinoma
}

\author{
${\text { GANG } \mathrm{LI}^{1 *} \text {, YANPING ZHONG }}^{1^{*}}$, QINGRONG SHEN ${ }^{4 *}$, YI ZHOU $^{1}$, XIAOFANG DENG $^{2}$, \\ CUIPING $\mathrm{LI}^{2}$, JIAGUI CHEN ${ }^{2}$, YING ZHOU ${ }^{2}$ and $\mathrm{MIN} \mathrm{HE}^{2,3}$ \\ ${ }^{1}$ Medical Scientific Research Center, Guangxi Medical University, Nanning, Guangxi 530021; ${ }^{2}$ School of Public Health, \\ Guangxi Medical University, Nanning, Guangxi 530021; ${ }^{3}$ Key Laboratory of High-Incidence-Tumor Prevention and Treatment \\ (Guangxi Medical University), Ministry of Education, Nanning, Guangxi 530021; ${ }^{4}$ Department of Pharmacy, The People's \\ Hospital of Guangxi Zhuang Autonomous Region, Nanning, Guangxi Zhuang Autonomous Region 530021, P.R. China
}

Received October 26, 2016; Accepted December 19, 2016

DOI: $10.3892 /$ ijo.2017.3837

\begin{abstract}
Never in mitosis gene A (NIMA)-related kinase 2 (NEK2) is a microtubule-associated protein that regulates spindle assembly in human cells and is overexpressed in various malignancies. However, the role of NEK 2 in hepatocellular carcinoma (HCC) remains undetermined. We performed RNA-seq of the HCC cell line SMMC-7721 and the normal liver cell line HL-7702 using the Ion Proton System. NEK2 expression was detected using quantitative reverse transcription polymerase chain reaction in two cell lines and 5 matched HCC and adjacent non-tumorous liver tissues. The correlation between survival and NEK2 expression was analyzed in 359 patients with HCC using RNASeqV2 data available from The Cancer Genome Atlas (TCGA) website (https://tcga-data. nci.nih.gov/tcga/). The expression of NEK2, phospho-AKT and MMP-2 was evaluated by immunohistochemistry in 63 cases of HCC and matched adjacent non-tumorous liver tissues. Relationships between protein expression and clinicopathological parameters were assessed, and the correlations between NEK2 with phospho-AKT and MMP-2 expressions were evaluated. A total of 610 differentially expressed genes (DEGs) were revealed in the transcriptome comparison, 297 of which were upregulated and 313 were downregulated in HCC. NEK2, as the most obviously different DEG in cells and tissues from the RNA-seq data, was listed as an HCC candidate biomarker for further verification. NEK2 was overexpressed in HCC cells and tissues $(\mathrm{P}=0.002, \mathrm{P}=0.013)$ and $\mathrm{HCC}$ patients with a high expression of NEK2 had a poor prognosis $(\mathrm{P}=0.0145)$. Clinical analysis indicated that the overexpression of NEK2 in HCC was significantly correlated with diolame
\end{abstract}

Correspondence to: Dr Min He, School of Public Health, Guangxi Medical University, Nanning, Guangxi 530021, P.R. China

E-mail: hemin@gxmu.edu.cn

*Contributed equally

Key words: NEK2, prognostic biomarker, hepatocellular carcinoma complete $(\mathrm{P}<0.001)$, tumor nodule number $(\mathrm{P}=0.012)$ and recurrence $(\mathrm{P}=0.004)$. NEK2 expression was positively correlated with the expression of phospho-AKT $(\mathrm{r}=0.883, \mathrm{P}<0.01)$ and MMP-2 ( $\mathrm{r}=0.781, \mathrm{P}<0.01)$. Overexpression of NEK2 was associated with clinicopathological characteristics and poor patient outcomes, suggesting that NEK2 serves as a prognostic biomarker for HCC. Alteration of NEK2 protein levels may contribute to invasion and metastasis of HCC, which may occur through activation of AKT signaling and promotion of MMP-2 expression.

\section{Introduction}

Hepatocellular carcinoma (HCC) is the sixth most prevalent type of cancer and the second leading cause of cancer-related deaths worldwide (1-3). Epidemiological studies show that the incidence of HCC is markedly varied across geographical regions, ancestry groups and between genders (4). HCC incidence is highest in East Asia and Africa and rapid increases in prevalence have occurred in Western countries (5). The typical characteristics of HCC include fast infiltrating growth, abnormal cell differentiation, high-grade malignancy, early metastasis and poor prognosis $(6,7)$. Therefore, identifying novel and reliable biomarkers to identify, predict and treat HCC are urgently needed.

NEK2 [NIMA (never in mitosis gene A)-related expressed kinase 2], a serine/threonine centrosomal kinase, plays a critical role in regulating the cell cycle and mitosis by centrosome splitting during the cell division process (8). Uncontrolled NEK2 activity can result in chromosome instability (CIN) and abnormal chromosome content $(9,10)$. NEK2 overexpression has been demonstrated in several types of human cancers, such as breast (11-14), prostate (11), non-Hodgkin lymphoma (15) and colorectal cancer (16). Additionally, some reports have indicated NEK2 as a potential biomarker for pancreatic ductal adenocarcinoma and non-small cell lung cancer prognosis $(17,18)$, but NEK2 has rarely been investigated with regard to HCC.

With the advancements in the next generation of sequencing technologies, RNA-seq has become a powerful tool for deciphering global gene expression patterns, including an 
unprecedented capability to discover novel genes, alternative transcript variants, chimeric transcripts, expressed sequence variants and allele-specific expressions (19-22). RNA-seq has expanded the study of cancer transcriptomics in the areas of gene expression, chimeric events and alternative splicing in search of novel biomarkers for the disease (23).

By integrating the RNA-seq data for the cells in the present study and the tissues in the study by Huang et al (24), we found that NEK2 expression was significantly upregulated and associated with a poor prognosis in patients with HCC. Therefore, NEK 2 may be a very promising prognostic biomarker for predicting HCC. Furthermore, the possible mechanisms responsible for NEK2 overexpression in HCC also were investigated.

\section{Materials and methods}

Patient information. A total of 63 patients who were diagnosed with HCC and treated with partial liver resection surgery at the Affiliated Tumor Hospital of Guangxi Medical University from 2010 to 2013 were enrolled in this study. These patients included 52 males and 11 females, with a mean age of 47.86 years (range, 28-71 years) at the time of the operation. The patients were pathologically diagnosed with HCC of histological grade II $(n=28)$, grade III $(n=20)$ and grade IV $(n=15)$ according to the modified nuclear grading scheme outlined by the Edmondson and Steiner system. A summary of the patient characteristics and the pathological characteristics is presented in Table I. For validation using quantitative reverse transcription-polymerase chain reaction (qRT-PCR), HCC tissues and matched adjacent non-tumorous liver tissues from 5 different HCC patients (aged 42-68 years) were provided by the First Affiliated Hospital of Guangxi Medical University in 2014. No prior treatments (including chemotherapy or radiotherapy) were conducted before the liver resection surgery. This study was approved by the Ethics Committee of the Guangxi Medical University. All patients provided written informed consent in order to participate in this study.

Tissues samples and cell lines. All HCC tissues and matched adjacent non-tumorous liver tissues were obtained immediately after hepatectomy and were frozen in liquid nitrogen and stored at $-80^{\circ} \mathrm{C}$ or collected in $10 \%$ formalin and embedded in paraffin for histopathological analysis. The human HCC cell line SMMC-7721 and primary human normal liver cell line HL-7702 were purchased from the Committee on Type Culture Collection of Chinese Academy of Sciences (Shanghai, China). All cell lines were maintained under recommended culture conditions. Cells were incubated in a $37^{\circ} \mathrm{C}$ humidified incubator containing $5 \% \mathrm{CO}_{2}$.

Transcriptome sequencing. For whole transcriptome

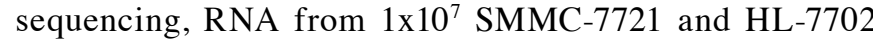
cells was extracted using the TRIzol reagent kit (Invitrogen, Waltham, MA, USA) and then quantified using NanoDrop 2000 (Thermo Fisher Scientific, Waltham, MA, USA). The whole transcriptome RNA-seq procedure was performed using the Ion Total RNA-Seq kit, the Ion PI ${ }^{\mathrm{TM}}$ Chip kit, the Ion $\mathrm{PI}^{\mathrm{TM}}$ Template OT2 200 kit, and the Ion PI ${ }^{\mathrm{TM}}$ Sequencing 200 kit based on the protocols of Life Technologies Corp. (Waltham,
MA, USA). In brief, mRNA was purified using oligo-dT beads from $100 \mu \mathrm{g}$ of total RNAs for each sample and then fragmented. The cleaved RNA fragments were reverse-transcribed into First-Strand cDNA, followed by Second-Strand cDNA synthesis. Then, a single 'A' base was added to the cDNA fragments at the $3^{\prime}$ end. The cDNAs were ligated to adapters and enriched by polymerase chain reaction (PCR) to generate the final cDNA library. After amplifying the sequencing template, RNA-seq was performed using the Ion Proton System (Life Technologies) with the standard protocol.

$q R T-P C R$. Total RNA was extracted from cell lines and liver specimens using the TRIzol reagent kit (Invitrogen) according to the manufacturer's instructions. To avoid any DNA contamination, isolated RNA was treated with RNase-free DNase I (Invitrogen) and quantified by NanoDrop 2000 (Thermo Fisher Scientific). The RNA samples were measured using optical density at $260 \mathrm{~nm}$ and then reverse-transcribed into cDNA using the M-MLV First-Strand system for the qRT-PCR kit (Invitrogen) according to the manufacturer's protocols. qRT-PCR was performed using the FastStart Universal SYBR-Green Master (Roche Diagnostics, Shanghai, China) and repeated three times in an ABI 7500 system. The primer sequences used to detect mRNA were as follows: NEK2, forward 5'-CTTCCCGGGCTGAGGACTAT-3' and reverse 5'-TCAGCTTCTGTCATGGAGCC-3'; $\beta$-actin, forward 5'-GG GAAATCGTGCGTGACAT-3' and reverse 5'-CTGGAAGG TGGACAGCGAG-3'.

The PCR cycling conditions were as follows: initial melting at $95^{\circ} \mathrm{C}$ for $30 \mathrm{sec}$, followed by 40 cycles at $95^{\circ} \mathrm{C}$ for $5 \mathrm{sec}$ and $60^{\circ} \mathrm{C}$ for $64 \mathrm{sec}$. Analysis of the melting curve for the primers was conducted to confirm the specificity of the PCR product, and the threshold cycle $(\mathrm{Ct})$ value for triplicate reactions was averaged. The relative expression of NEK2 mRNA for each sample was calculated as follows: $\Delta \mathrm{Ct}=\mathrm{Ct}$ (sample) $-\mathrm{Ct}$ ( $\beta$-actin), $\Delta \Delta \mathrm{Ct}$ (sample) $=\Delta \mathrm{Ct}$ (sample) $-\Delta \mathrm{Ct}$ (calibrator) . The fold changes in mRNA were calculated through relative quantification $\left(2^{-\Delta \Delta C t}\right)$.

Immunohistochemistry and immunohistochemical assessment. Immunohistochemical studies on NEK2, phospho-AKT and MMP-2 were performed on formalin-fixed, paraffinembedded tissue sections obtained from the aforementioned patients with HCC and were performed according to the standard procedures. Sections were cut at a thickness of $5 \mu \mathrm{m}$ and heated in a $60^{\circ} \mathrm{C}$ oven. Briefly, tissue sections were deparaffinized, rehydrated and boiled in $0.01 \mathrm{~mol} / 1$ sodium citrate buffer (pH 6.0) in a microwave oven for 10 min for antigen epitope retrieval. Endogenous peroxidase was blocked with $0.3 \%$ hydrogen peroxide for $10 \mathrm{~min}$. Then, the sections were blocked for 30 min using $10 \%$ normal goat serum and were separately incubated with the primary antibodies directed against NEK2 (ab55550, 1:1,000 dilution; Abcam, Cambridge, MA, USA), phospho-AKT (Ser473) (\#4060, 1:50 dilution; Cell Signaling Technology) and MMP-2 (ab86607 1:500, dilution; Abcam) at $37^{\circ} \mathrm{C}$ for $3 \mathrm{~h}$. After washing, the sections were incubated for $30 \mathrm{~min}$ with biotinylated secondary antibody (Envision $^{\mathrm{TM}}$ Detection kit; Gene Tech, Shanghai, China) at $37^{\circ} \mathrm{C}$. The staining of the tissue sections was performed using the streptavidin-biotin-peroxidase complex for NEK2, 
Table I. Relationships between NEK2, phospho-AKT and MMP-2 expression and clinicopathological variables of HCC.

\begin{tabular}{|c|c|c|c|c|c|c|c|}
\hline \multirow[b]{2}{*}{ Characteristics } & \multirow[b]{2}{*}{$\mathrm{n}$} & \multicolumn{2}{|c|}{ NEK2 (lg IOD) } & \multicolumn{2}{|c|}{ P-AKT (lg IOD) } & \multicolumn{2}{|c|}{ MMP-2 (lg IOD) } \\
\hline & & Mean \pm SD & P-value & Mean \pm SD & P-value & Mean \pm SD & P-value \\
\hline Age (years) & & & 0.321 & & 0.551 & & 0.415 \\
\hline$\leq 50$ & 36 & $3.64 \pm 0.86$ & & $2.98 \pm 0.73$ & & $4.47 \pm 0.56$ & \\
\hline$>50$ & 27 & $3.72 \pm 0.90$ & & $3.08 \pm 0.71$ & & $4.56 \pm 0.40$ & \\
\hline Gender & & & 0.285 & & 0.805 & & 0.450 \\
\hline Male & 52 & $3.69 \pm 0.92$ & & $3.03 \pm 0.74$ & & $4.45 \pm 0.45$ & \\
\hline Female & 11 & $3.60 \pm 0.66$ & & $2.97 \pm 0.55$ & & $4.41 \pm 0.70$ & \\
\hline Serum AFP (ng/ml) & & & 0.076 & & 0.164 & & 0.497 \\
\hline$\leq 25$ & 28 & $3.75 \pm 0.91$ & & $3.20 \pm 0.72$ & & $4.55 \pm 0.54$ & \\
\hline $25-400$ & 6 & $3.77 \pm 0.65$ & & $2.93 \pm 0.62$ & & $4.36 \pm 0.49$ & \\
\hline$>400$ & 29 & $3.59 \pm 0.89$ & & $2.86 \pm 0.73$ & & $4.51 \pm 0.44$ & \\
\hline Tumor size $(\mathrm{cm})$ & & & $0.024^{\mathrm{a}}$ & & $0.041^{\mathrm{a}}$ & & $0.013^{\mathrm{a}}$ \\
\hline$\leq 10$ & 46 & $3.73 \pm 0.89$ & & $3.13 \pm 0.75$ & & $4.65 \pm 0.41$ & \\
\hline$>10$ & 17 & $3.53 \pm 0.84$ & & $2.78 \pm 0.60$ & & $4.29 \pm 0.72$ & \\
\hline Portal vein thrombosis & & & 0.428 & & 0.282 & & 0.628 \\
\hline Presence & 20 & $3.62 \pm 0.80$ & & $3.14 \pm 0.80$ & & $4.46 \pm 0.41$ & \\
\hline Absence & 43 & $3.70 \pm 0.92$ & & $2.95 \pm 0.67$ & & $4.52 \pm 0.53$ & \\
\hline Diolame complete & & & $0.000^{\mathrm{c}}$ & & 0.948 & & $0.009^{\mathrm{b}}$ \\
\hline Yes & 28 & $3.51 \pm 0.97$ & & $3.03 \pm 0.75$ & & $4.41 \pm 0.56$ & \\
\hline No & 35 & $3.79 \pm 0.79$ & & $3.02 \pm 0.69$ & & $4.72 \pm 0.38$ & \\
\hline Tumor nodule number & & & $0.012^{\mathrm{a}}$ & & $0.046^{\mathrm{a}}$ & & $0.024^{\mathrm{a}}$ \\
\hline Solitary & 44 & $3.59 \pm 0.91$ & & $2.96 \pm 0.71$ & & $4.44 \pm 0.55$ & \\
\hline Multiple $(\geq 2)$ & 19 & $3.80 \pm 0.76$ & & $3.34 \pm 0.74$ & & $4.75 \pm 0.44$ & \\
\hline Edmondson grade & & & 0.121 & & 0.293 & & 0.855 \\
\hline II & 28 & $3.85 \pm 0.88$ & & $3.29 \pm 0.85$ & & $4.62 \pm 0.57$ & \\
\hline III & 20 & $3.97 \pm 0.53$ & & $3.02 \pm 0.35$ & & $4.54 \pm 0.44$ & \\
\hline IV & 15 & $3.61 \pm 0.90$ & & $2.80 \pm 0.57$ & & $4.50 \pm 0.39$ & \\
\hline Liver cirrhosis & & & 0.474 & & 0.728 & & 0.192 \\
\hline Yes & 53 & $3.68 \pm 0.84$ & & $3.01 \pm 0.71$ & & $4.54 \pm 0.50$ & \\
\hline No & 10 & $3.57 \pm 1.21$ & & $2.93 \pm 0.71$ & & $4.33 \pm 0.52$ & \\
\hline HBV-DNA & & & 0.435 & & 0.918 & & 0.315 \\
\hline Positive & 42 & $3.67 \pm 0.90$ & & $3.02 \pm 0.76$ & & $4.51 \pm 0.47$ & \\
\hline Negative & 21 & $3.73 \pm 0.79$ & & $3.04 \pm 0.56$ & & $4.62 \pm 0.32$ & \\
\hline Recurrence & & & $0.004^{\mathrm{b}}$ & & $0.045^{\mathrm{a}}$ & & 0.992 \\
\hline Yes & 32 & $3.98 \pm 0.77$ & & $3.17 \pm 0.53$ & & $4.57 \pm 0.48$ & \\
\hline No & 31 & $3.66 \pm 0.92$ & & $2.66 \pm 0.86$ & & $4.57 \pm 0.47$ & \\
\hline
\end{tabular}

${ }^{\mathrm{a}} \mathrm{P}<0.05 ;{ }^{\mathrm{b}} \mathrm{P}<0.01 ;{ }^{\mathrm{c}} \mathrm{P}<0.001$.

phospho-AKT and MMP-2. The complex was visualized with diaminobenzidine (DAB) and counterstained with hematoxylin. The sections were then dehydrated in a graded series of alcohol, cleared in xylene and mounted onto glass slides.

The staining was quantified by digital image analysis with Image-Pro Plus 6.0 software (Media Cybernetics, Silver Spring, MD, USA) according to the method developed by Xavie et al (25). Briefly, an area of interest in each section was first selected at x40 magnification, and 10 digital images at $1360 \times 1024$ pixel resolution and $\times 400$ magnification were captured using an AX-70 microscope equipped with a DP70 CCD camera (Olympus, Tokyo, Japan). Identical settings were used for each field. The measurement parameter was integrated optical density (IOD). Optical density was calibrated, and the area of interest was set as follows: hue, 0-30; saturation, 0-255; and intensity, 0-255. The values were then counted. 
The Cancer Genome Atlas (TCGA) data acquisition and survival analysis. The TCGA project provides multimodal data on 359 HCC cases, which can be acquired from the TCGA website (https://tcga-data.nci.nih.gov/tcga/). The dataset was searched for HCC cases based on the RNASeqV2 data. The expression value of the NEK2 gene was collected for each case and was divided into the highexpression and the low-expression groups using the cut-off point. Kaplan-Meier survival analysis was used to determine the survival differences between the high-expression and low-expression subgroups, with P-values calculated using the log-rank test.

Statistical analysis. Statistical analyses were performed with IBM SPSS Statistics 20.0 (IBM Corp., Armonk, NY, USA). Relationships between the expressions of NEK2, phosphoAKT and MMP-2 and the clinicopathological parameters were determined using the two-tailed unpaired Student's t-test. Significance among three groups was determined by analysis of variance (ANOVA) followed by the Duncan's new multiple range test. The correlations between the NEK 2 expression with phospho-AKT and MMP-2 expressions were studied using the Spearman's coefficient. Data are shown as the means \pm standard error of the means. $\mathrm{P}<0.05$ was considered to indicate statistically significant differences.

\section{Results}

Analysis of the cell RNA-seq data. RNA-seq of the HCC cell line SMMC-7721 and the normal liver cell line HL-7702 was performed using the Ion Proton System. On average, 78.1 million 96-bp-long sequencing reads and $7.5 \mathrm{G}$ of raw sequence data were obtained for samples sequenced on one lane. The normalized gene expression was measured as fragments per kilobase of transcript per million mapped reads (FPKM). To evaluate differential gene expression, the absolute value of the $\log 2$-transformed fold change (FC) $\geq 1$ and the q-values $<0.05$ were used as the criteria to determine the significance of gene expression differences. A total of 610 differentially expressed genes (DEGs) were revealed in the transcriptome comparison, 297 of which were upregulated and 313 were downregulated in HCC.

NEK2 was listed as an HCC candidate biomarker by integrated analysis. Huang et al (24) performed RNA-seq analyses of 10 matched pairs of cancerous and non-cancerous tissues from HCC patients on the Solexa/Illumina GAII platform. The results showed that a total of 1378 DEGs with 808 upregulation and 570 downregulation in HCC tissues compared with adjacent non-tumorous liver tissues. An integrated analysis was then performed on the RNA-seq data of the cells and tissues. As shown in the Venn diagram in Fig. 1A, 12 common differential genes were found between the HCC cells and the normal cells and between the HCC tissues and the adjacent tissues, 10 of which were upregulated and 2 were downregulated in HCC (Table II). Excitingly, NEK2 exhibited the most significant difference in expression of all the DEGs in the cell and tissue RNA-seq data and was therefore listed as an $\mathrm{HCC}$ candidate biomarker for further verification (Fig. 1B and C).
A

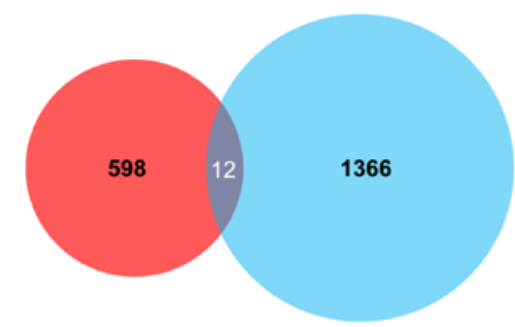

HCC cells vs. normal cells (610 DEGs)

HCC tissues vs. adjacent tissues (1378 DEGs)
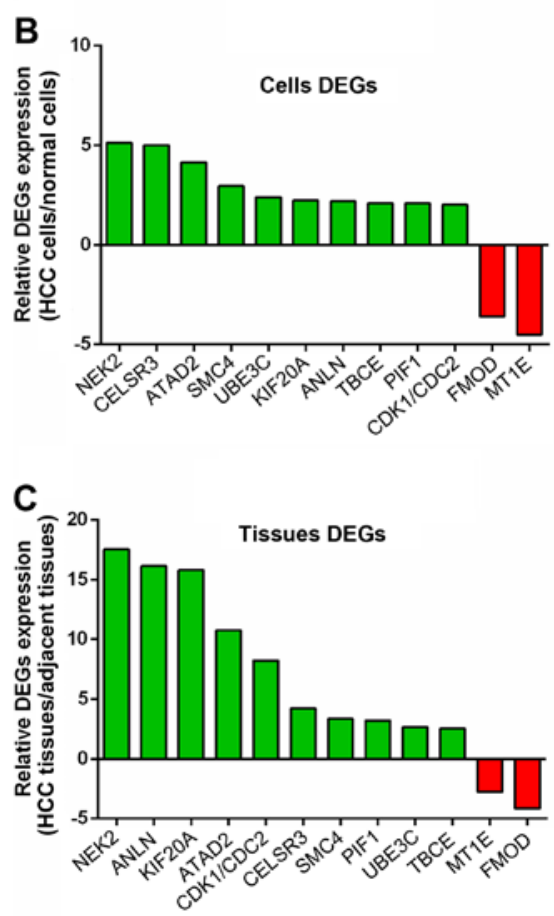

Figure 1. The common differential genes between cell DEGs and tissue DEGs. (A) Venn diagram of the overlaps between cell DEGs and tissue DEGs. The red circle represents 610 DEGs between HCC cells and normal liver cells. The blue circle represents 1378 DEGs between HCC tissues and adjacent non-tumorous liver tissues. There were 12 common differential genes between the two circles. (B) Relative expression of 12 common differential genes in HCC cell line SMMC-7721, when compared with normal liver cell HL-7702. (C) Relative expression of 12 common differential genes in HCC tissues, when compared with adjacent non-tumorous liver tissues.

The overexpression of NEK2 in HCC cells and tissues. NEK2 expression status in the HCC cell line SMMC-7721 and the primary HCC tissue samples was assessed using qRT-PCR. The NEK2 mRNA transcript level was $0.024 \pm 0.0026$ in the HCC cell line SMMC-7721, which was 1.71-fold higher than the NEK 2 mRNA level of $0.014 \pm 0.0003$ in the normal liver cell line HL-7702 ( $\mathrm{P}=0.002)$ (Fig. 2A), as evidenced by qRT-PCR analysis using $\beta$-actin as a loading control. In 5 matched HCC and adjacent non-tumorous liver tissue samples, the NEK2 mRNA level in the HCC tissues was $5.60 \pm 3.69$, which was 16.47-fold higher than the NEK2 mRNA level of $0.34 \pm 0.38$ in adjacent non-tumorous liver tissues ( $\mathrm{P}=0.013)$ (Fig. 2B).

The expression of NEK2 in 63 cases of HCC and matched adjacent non-tumorous liver tissues was examined using immunohistochemical staining. The NEK2 protein is mainly expressed in the cytoplasm, and we quantified its expression 
A

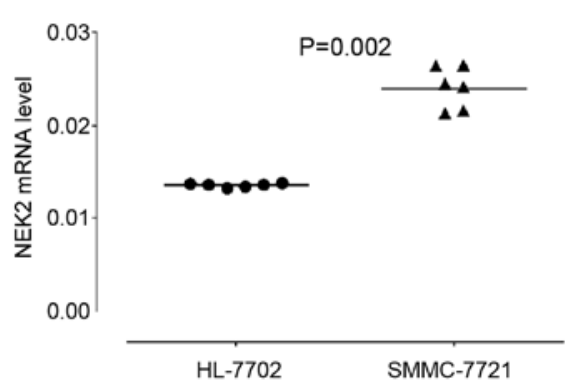

C

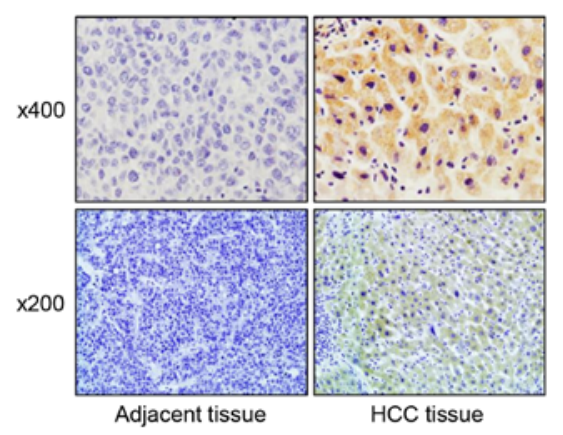

B

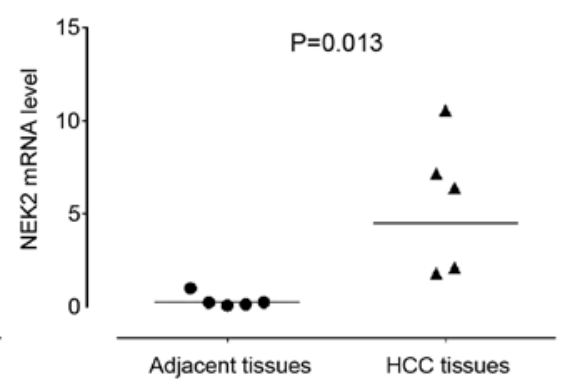

D

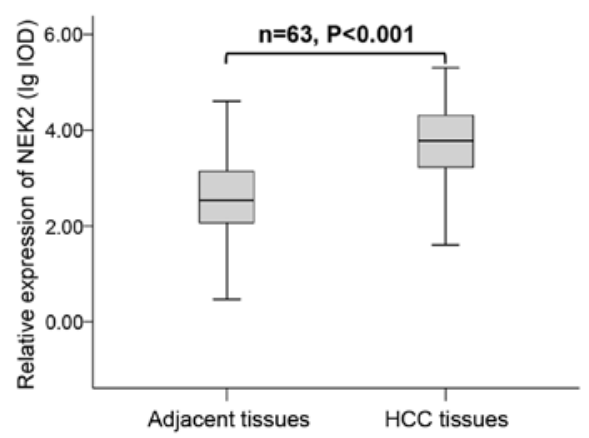

Figure 2. Expression of NEK2 in HCC cell lines and HCC tissues. (A) NEK2 mRNA expression in immortalized normal human liver cell line HL-7702 and HCC cell line SMMC-7721 using quantitative reverse transcription polymerase chain reaction $(\mathrm{qRT}-\mathrm{PCR})(\mathrm{n}=6$, $\mathrm{P}=0.002)$. (B) NEK2 mRNA expression in HCC tissues and matched adjacent non-tumorous liver tissues using qRT-PCR ( $\mathrm{n}=5, \mathrm{P}=0.013)$. (C) Immunohistochemical staining for NEK2 expression in HCC and adjacent non-tumorous liver tissues. Representative images (x200; x400) of positive staining in HCC tissues and negative staining in adjacent nontumorous liver tissues. (D) Immunohistochemical staining integrated optical density (IOD) value of NEK2 in HCC and adjacent non-tumorous liver tissues $(\mathrm{n}=63, \mathrm{P}<0.001)$.

Table II. List of common differential genes between cells DEGs and tissues DEGs.

\begin{tabular}{|c|c|c|c|c|c|}
\hline No. & $\begin{array}{c}\text { Gene } \\
\text { symbol }\end{array}$ & Regulation & $\begin{array}{c}\text { Cells } \\
(\mathrm{HCC} / \mathrm{N})\end{array}$ & $\begin{array}{l}\text { Tissues } \\
(\mathrm{HCC} / \mathrm{N})\end{array}$ & Gene description \\
\hline 1 & CELSR3 & Upregulation & 5.00 & 4.21 & Cadherin EGF LAG seven-pass G-type receptor 3 precursor \\
\hline 2 & UBE3C & Upregulation & 2.39 & 2.67 & Ubiquitin-protein ligase E3C \\
\hline 3 & ANLN & Upregulation & 2.18 & 18.13 & Actin-binding protein anillin \\
\hline 4 & KIF20A & Upregulation & 2.24 & 15.8 & Kinesin-like protein KIF20A \\
\hline 5 & SMC4 & Upregulation & 2.94 & 3.35 & Structural maintenance of chromosomes protein 4 \\
\hline 6 & TBCE & Upregulation & 2.08 & 2.53 & Tubulin-specific chaperone E \\
\hline 7 & NEK2 & Upregulation & 5.13 & 17.52 & Serine/threonine-protein kinase Nek2 \\
\hline 8 & PIF1 & Upregulation & 2.08 & 3.21 & ATP-dependent DNA helicase PIF1 \\
\hline 9 & ATAD2 & Upregulation & 4.14 & 10.74 & ATPase family AAA domain-containing protein 2 \\
\hline 10 & $\mathrm{CDK} 1 / \mathrm{CDC} 2$ & Upregulation & 2.00 & 8.22 & Cell division control protein 2 homolog \\
\hline 11 & FMOD & Downregulation & 0.28 & 0.24 & Fibromodulin precursor \\
\hline 12 & MT1E & Downregulation & 0.22 & 0.36 & Metallothionein-1E \\
\hline
\end{tabular}

HCC/N, hepatocellular carcinoma/non-tumor.

using Image-Pro Plus 6.0 digital image analysis software. We found that NEK2 expression presented as positive staining in $\mathrm{HCC}$ tissues and negative staining in adjacent non-tumorous liver tissues (Fig. 2C). Furthermore, the expression of NEK2 protein was significantly higher in the HCC tissues than in the adjacent non-tumorous liver tissues $(\mathrm{P}<0.001)$ (Fig. 2D).
NEK2 overexpression is significantly associated with poor prognosis in $\mathrm{HCC}$. To evaluate the clinical significance of NEK2 overexpression, the correlation between the survival and the expression of NEK2 in the cases of 359 patients with HCC was analyzed using RNASeqV2 data available from The Cancer Genome Atlas (TCGA) website. According to 

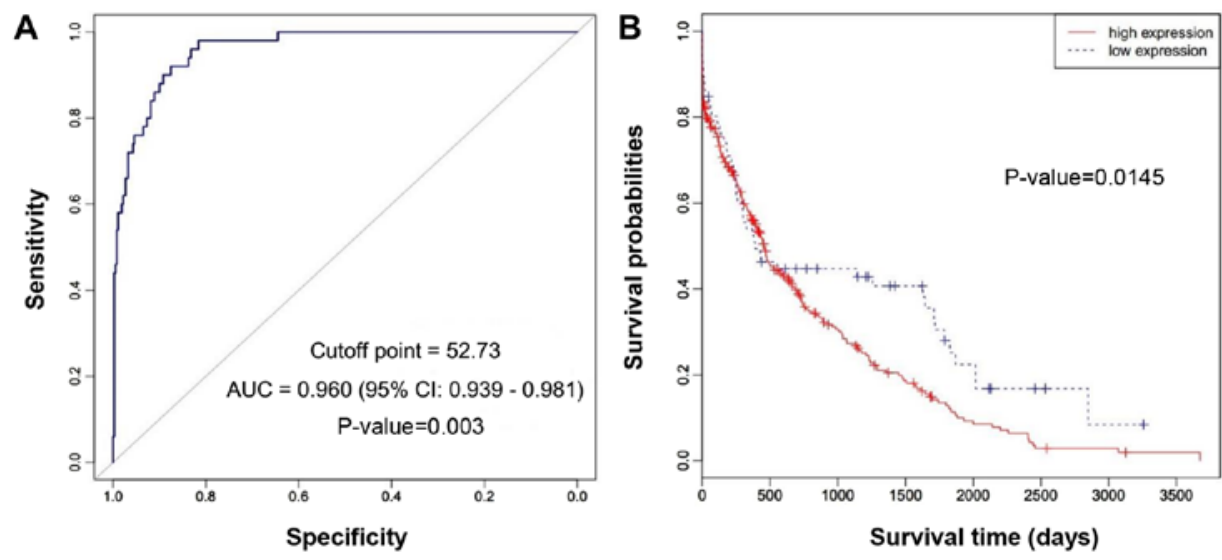

Figure 3. ROC and Kaplan-Meier curves for NEK2 expression in TCGA HCC RNAseq dataset. (A) ROC curve analysis to determine the cut-off point for the high expression of NEK2. The area under curve (AUC) was 0.960 (95\% CI, 0.939-0.981), and the cut-off point of NEK2 for high expression was 52.73. (B) Kaplan-Meier curve for HCC patients in high-expression $(\mathrm{n}=66)$ and low-expression $(\mathrm{n}=293)$ groups segregated by the cut-off point. HCC patients with a high expression of NEK2 had a poor prognosis (log-rank test, $\mathrm{P}=0.0145)$.
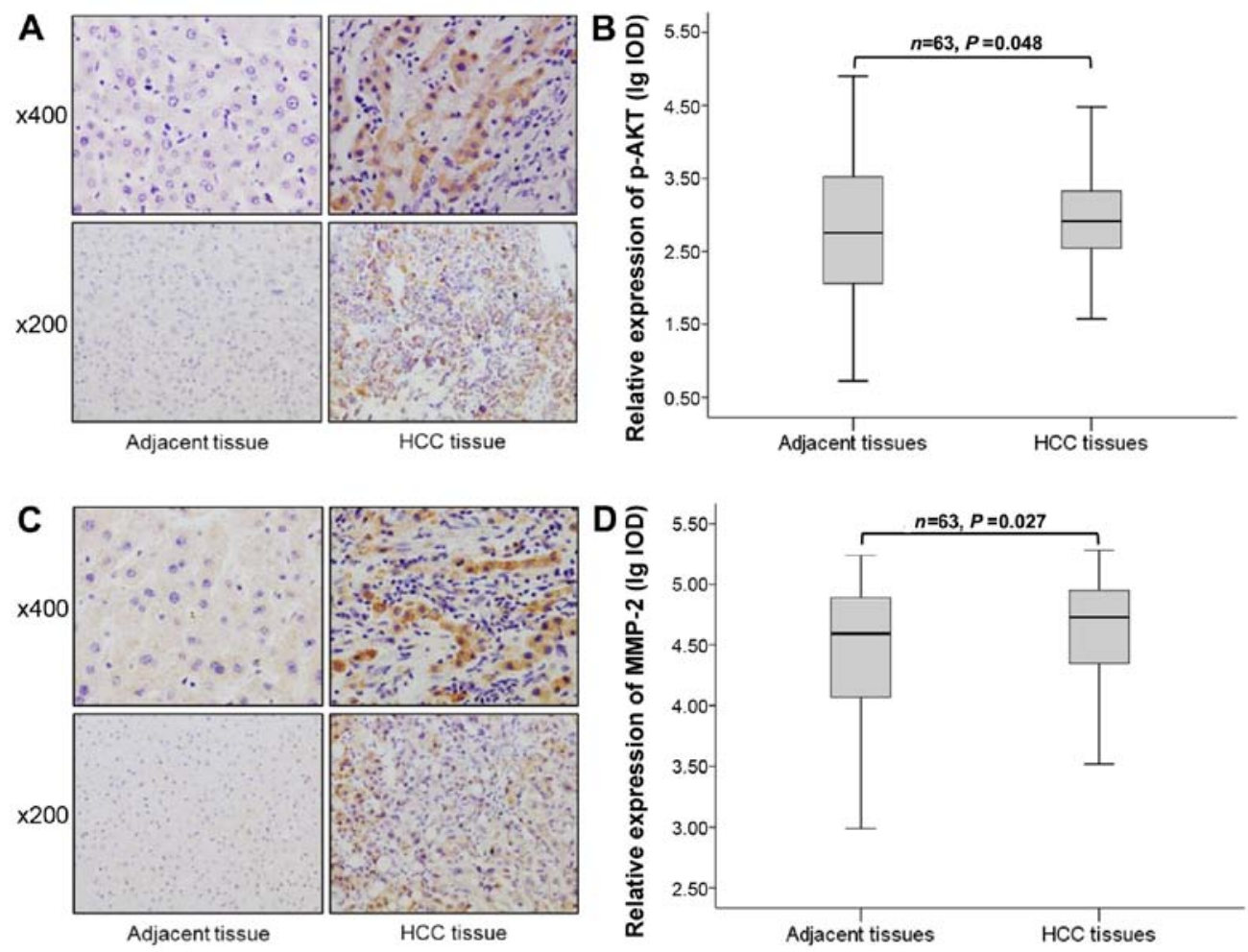

Figure 4. Immunohistochemical staining of phospho-AKT and MMP-2 expression in HCC and corresponding tumor-adjacent tissues. (A) Immunohistochemical staining for phospho-AKT expression in HCC and adjacent non-tumorous liver tissues. Representative images (x200; $\mathrm{x} 400$ ) of positive staining in HCC tissues and negative staining in adjacent non-tumorous liver tissues. (B) Immunohistochemical staining integrated optical density (IOD) value of phospho-AKT in $\mathrm{HCC}$ and adjacent non-tumorous liver tissues $(\mathrm{n}=63, \mathrm{P}=0.0048)$. (C) Immunohistochemical staining for MMP-2 expression in HCC and adjacent non-tumorous liver tissues. Representative images (x200; x400) of positive staining (right) in HCC tissues and negative staining (left) in adjacent non-tumorous liver tissues. (D) Immunohistochemical staining integrated optical density (IOD) value of MMP-2 in HCC and adjacent non-tumorous liver tissues $(\mathrm{n}=63$, $\mathrm{P}=0.027)$.

the receiver operating characteristic (ROC) curve, we defined RPKM=52.73 as the cut-off point to distinguish HCC patients with high and low NEK2 expression levels (Fig. 3A). The sensitivity and specificity of NEK2 in the diagnosis of HCC were 0.98 and 0.82 , respectively. Kaplan-Meier survival analysis of HCC patients was performed based on the expression levels of NEK2. The results revealed that HCC patients with a high expression of NEK2 had a poor prognosis (log-rank test, $\mathrm{P}=0.0145$ ) (Fig. 3B).
Expressions of phospho-AKT and MMP-2 were increased in HCC clinical samples. Previous studies suggested that the overexpression of NEK2 promotes activation of AKT, a potent and critical oncogene for a variety of malignancies $(26,27)$. Moreover, the PI3K/AKT signaling pathway plays an important role in upregulating MMP expression (28). Therefore, to further investigate the relationship between the NEK2 expression and the expression of phospho-AKT and MMP-2 in HCC, we assessed the expression of phospho-AKT and MMP-2 in 

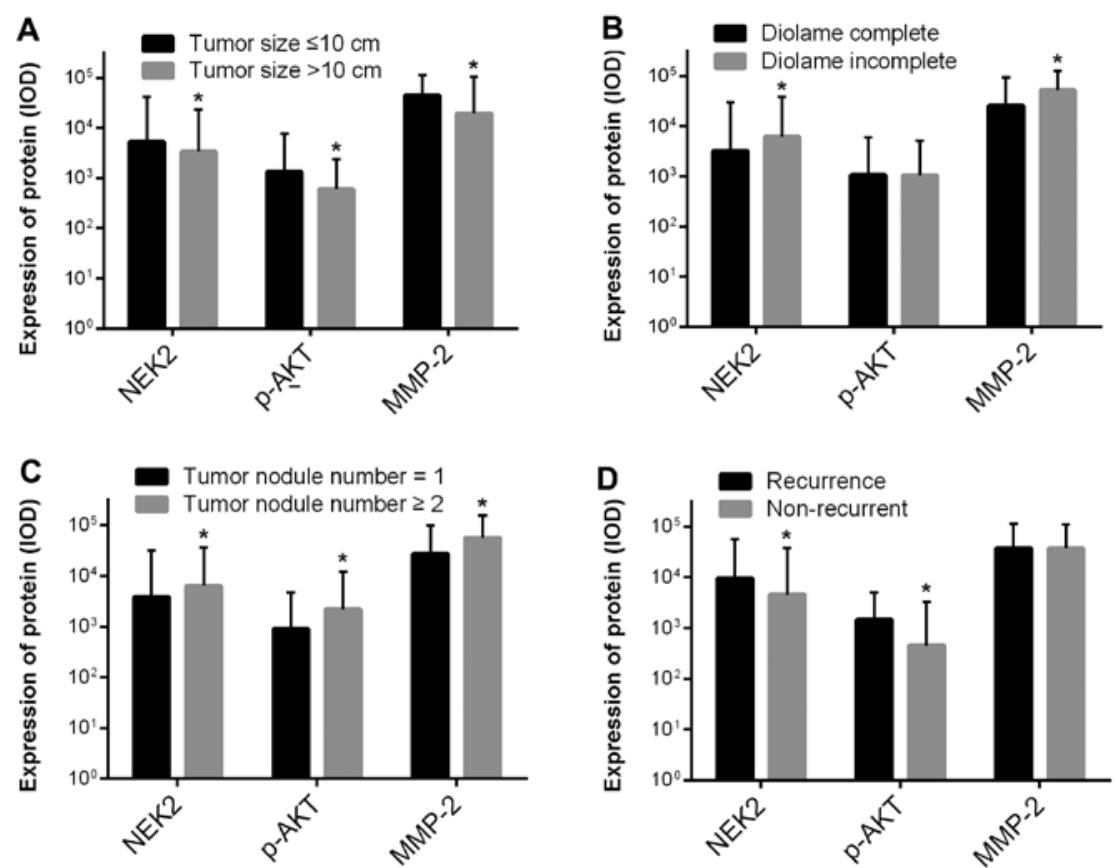

Figure 5. Relationships between NEK2, phospho-AKT, and MMP-2 expression and clinicopathological features in HCC. (A) NEK2, phospho-AKT and MMP-2 expression in the HCC tumor size $\leq 10 \mathrm{~cm}$ group was 1.58 -fold $(\mathrm{P}=0.024), 2.24$-fold $(\mathrm{P}=0.041)$ and 2.29 -fold $(\mathrm{P}=0.013)$ higher, respectively, than that in the HCC tumor size $>10 \mathrm{~cm}$ group. (B) NEK2 and MMP-2 expression in the HCC diolame incomplete group was 1.91-fold $(\mathrm{P}<0.001)$ and 2.04-fold $(\mathrm{P}=0.009)$ higher, respectively, than that in the HCC diolame complete group, but no obvious change was observed in phospho-AKT expression in the HCC diolame incomplete group ( $\mathrm{P}=0.948)$. (C) NEK2, p-AKT and MMP-2 expression in the HCC multinodular group was 1.62-fold ( $\mathrm{P}=0.012)$, 2.40-fold ( $\mathrm{P}=0.046)$, and 2.04-fold ( $\mathrm{P}=0.024)$ higher, respectively, than that in the HCC uninodular group. (D) NEK2 and p-AKT expression in the HCC recurrence group was 2.09 -fold $(\mathrm{P}=0.004)$ and 3.24-fold $(\mathrm{P}=0.045)$ higher, respectively, than that in the HCC non-recurrence group, but no obvious change was observed in MMP-2 expression in the HCC non-recurrence group $(\mathrm{P}=0.992)$.

63 cases of HCC and matched adjacent non-tumorous liver tissues using immunohistochemical staining. The staining for phospho-AKT and MMP-2 was mostly positive in the cytoplasm of the tumor cells (Fig. 4A and C). We found that the expression of phospho-AKT and MMP-2 was increased in $\mathrm{HCC}$ tissues compared with matched adjacent non-tumorous liver tissues ( $\mathrm{P}=0.048$ and 0.027 ) (Fig. 4B and $\mathrm{D})$.

Relationships between the expression of NEK2, phospho$A K T$, and MMP-2 and clinicopathological parameters. Table I summarizes the relationships between the expression of NEK2, phospho-AKT and MMP-2 and the clinicopathological parameters of patients with $\mathrm{HCC}$, including patient age, gender, AFP level, tumor size, portal vein thrombosis, diolame complete, tumor nodule number, Edmondson grade, cirrhosis, HBV DNA and recurrence. The results showed that NEK2, phospho-AKT and MMP-2 expression in the HCC tumor size $\leq 10 \mathrm{~cm}$ group was 1.58 -fold $(\mathrm{P}=0.024), 2.24$-fold $(\mathrm{P}=0.041)$, and 2.29-fold $(\mathrm{P}=0.013)$ higher, respectively, than that in the HCC tumor size $>10 \mathrm{~cm}$ group (Fig. 5A). NEK2 and MMP-2 expression in the HCC diolame incomplete group was 1.91-fold $(\mathrm{P}<0.001)$ and 2.04-fold $(\mathrm{P}=0.009)$ higher, respectively, than that in the HCC diolame complete group, but no obvious change was observed in phospho-AKT expression in the HCC diolame incomplete group $(\mathrm{P}=0.948)$ (Fig. 5B). NEK2, p-AKT and MMP-2 expression in the HCC multinodular group was 1.62 -fold $(\mathrm{P}=0.012), 2.40$-fold $(\mathrm{P}=0.046)$, and 2.04-fold $(\mathrm{P}=0.024)$ higher, respectively, than that in the HCC uninodular group (Fig. 5C). NEK2 and p-AKT expression in the HCC recurrence group was 2.09-fold $(\mathrm{P}=0.004)$ and 3.24-fold $(\mathrm{P}=0.045)$ higher, respectively, than that in the HCC non-recurrence group, but no obvious change was observed in MMP-2 expression in the HCC non-recurrence group ( $\mathrm{P}=0.992)$ (Fig. 5D).

Positive correlation between NEK2 expression with phospho$A K T$ and MMP-2 expression. Additionally, to explore whether the NEK2 expression level was correlated with phospho-AKT and MMP-2 expression, the protein expressions of phosphoAKT and MMP-2 were examined in 63 cases of HCC tissues using immunohistochemical staining. Therefore, the correlations between NEK2 expression with phospho-AKT and MMP-2 expression were analyzed. The results showed that there was indeed evident positive correlation between the protein expression level of NEK 2 and phospho-AKT $(r=0.883$, $\mathrm{P}<0.01$ ) (Fig. 6A). Notably, a significant positive correlation was observed between the protein expression levels of NEK2 and MMP-2 (r=0.781, P<0.01) (Fig. 6B).

\section{Discussion}

NEK2 is a serine-threonine protein kinase of the NIMArelated kinase family that localizes to the centrosomes, which are the microtubule-organizing centers of a cell that regulate its separation (8). The NIMA-related kinase (Nek) family consists of eleven members (NEKs 1-11) (29). In humans, NEK2 exhibits the greatest sequence identity to NIMA (8). In the process of cell division, NEK 2 promotes centrosome splitting at the beginning of mitosis by the phosphorylation of multiple linker components (30). In addition to centrosome 

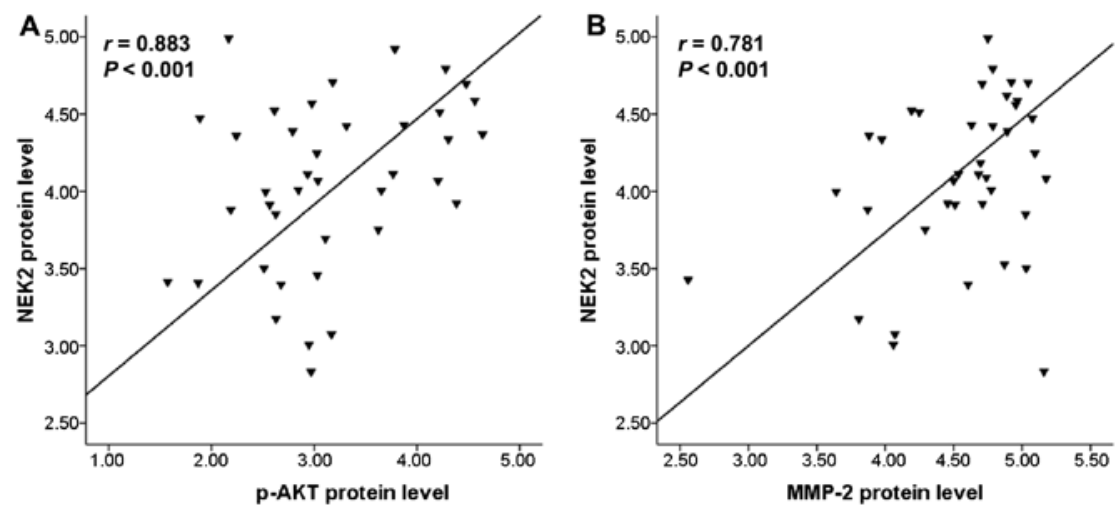

Figure 6. NEK2 expression and correlation with phospho-AKT and MMP-2 in HCC tissues. (A) Significant positive correlation between NEK2 expression and phospho-AKT expression ( $r=0.883$; $\mathrm{P}<0.001$ ). (B) Significant positive correlation between NEK2 expression and MMP-2 expression $(\mathrm{r}=0.781$; $\mathrm{P}<0.001)$.

separation, NEK2 also regulates the microtubule organization capacity of the centrosome $(31,32)$. Recent studies have shown that an elevated expression of NEK2 induces abnormal tumor proliferation and drug resistance in breast and ovarian cancers $(13,33,34)$. Furthermore, the significant upregulation of NEK2 has been demonstrated to be associated with the progression and poor prognosis of a series of malignant tumors originating in different organs and tissues, such as colorectal carcinoma (35), breast carcinoma (36) and myeloma (27).

However, whether NEK2 expression is elevated and associated with the clinicopathological features and prognosis of HCC remains unclear. In this study, we first assessed the expression of NEK2 in cells and tissues and found that NEK2 expression was significantly upregulated in HCC cells and tissues. Additionally, we examined the expression of NEK2 in a relatively large population of patients diagnosed with HCC and correlated it with the clinicopathological parameters and prognosis to verify whether this biomarker could predict HCC outcomes. The present study revealed that the overexpression of NEK2 was significantly correlated with diolame complete, tumor nodule number and recurrence. Thus, these results strongly confirm the intriguing possibility that the alteration of NEK2 protein levels may contribute to the invasion and metastasis of HCC.

Furthermore, Kaplan-Meier survival curve analysis demonstrated that HCC patients with a high expression of NEK2 had a poor prognosis, suggesting that NEK2 may be a potential prognostic factor for HCC patients. Therefore, NEK2 can be used as a novel prognostic biomarker to identify, distinguish, and predict HCC. The main limitation of this analysis is that, due to clinical covariates on HCC cases on TCGA website are not available, the multivariate Cox's regression survival model cannot be performed to assess the relative contribution of the risk group when assessed after adjusting for clinical variables.

Despite the important role of NEK2 in centrosome regulation and spindle formation, the mechanism of the abnormal expression and regulation of NEK2 remains unclear. Further studies are needed to clarify the mechanism that underlies the role of NEK2. Previous studies suggested that NEK2 may be involved in tumor progression through the influence of other tumor pathways. The elevation of NEK 2 contributes to the activation of the PI3K/AKT signaling pathway, a potent and critical oncogene for a variety of malignancies $(26,27)$. Moreover, the PI3K/AKT signaling pathway plays an important role in upregulating MMP expression (28), and aberrant AKT signaling could promote cell proliferation in HCC cells (37). Therefore, we sought to verify whether such a mechanism may contribute to HCC progression induced by NEK2. In this study, we found that the expression level of phospho-AKT and MMP-2 proteins was increased in HCC and positively correlated with NEK2, which indicated that overexpression of NEK2 may result in the high expression of phospho-AKT and MMP-2 proteins in HCC.

Furthermore, the AKT signaling pathway has been reported to play a key role in HCC cell invasion and metastasis by promoting the expression of MMP-2 (37), which is a key factor in HCC invasion and metastasis (38). The expression level of MMP-2 significantly reflects the aggressiveness of malignant tumor cells and is associated with poor prognosis in multiple tumor types (37). Combining the above findings with our results, we suggest that the invasion and metastasis effect of NEK2 in HCC may occur through the activation of AKT signaling and promotion of MMP-2 expression.

In conclusion, our data revealed that high expression of the NEK2 protein was common in HCC tissue samples and cultured hepatoma cell lines and was significantly associated with poor prognosis and unfavorable clinicopathological factors in HCC. Moreover, the NEK2 expression level was positively correlated with phospho-AKT and MMP-2 expression. Our results suggest that NEK2 is important for the progression, migration and invasion of $\mathrm{HCC}$ and may be a novel prognostic biomarker for HCC.

\section{Acknowledgements}

The present study was supported by the Guangxi Natural Science Foundation (grant nos. 2015GXNSFBA139162 and AB16380351), the National Natural Science Foundation of China (grant nos. 81260445 and 30960332), the Program of Key Laboratory of High-Incidence-Tumor Prevention and Treatment (Guangxi Medical University), the Ministry of Education, China (grant nos. GK2014-ZZ04 and GK2015-ZZ01), the Science and Technology Department of Nanning (grant no. 20151266), and the Innovation Project of Guangxi Graduate Education. 


\section{References}

1. Jemal A, Bray F, Center MM, Ferlay J, Ward E and Forman D: Global cancer statistics. CA Cancer J Clin 61: 69-90, 2011.

2. Kim HY and Park JW: Clinical trials of combined molecular targeted therapy and locoregional therapy in hepatocellular carcinoma: Past, present, and future. Liver Cancer 3: 9-17, 2014.

3. Njei B, Rotman Y, Ditah I and Lim JK: Emerging trends in hepatocellular carcinoma incidence and mortality. Hepatology 61: 191-199, 2015.

4. El-Serag HB: Epidemiology of viral hepatitis and hepatocellular carcinoma. Gastroenterology 142: 1264-1273.e1, 2012.

5. Forner A, Llovet JM and Bruix J: Hepatocellular carcinoma Lancet 379: 1245-1255, 2012.

6. Lazarevich NL, Cheremnova OA, Varga EV, Ovchinnikov DA, Kudrjavtseva EI, Morozova OV, Fleishman DI, Engelhardt NV and Duncan SA: Progression of HCC in mice is associated with a downregulation in the expression of hepatocyte nuclear factors. Hepatology 39: 1038-1047, 2004.

7. Tanaka S and Arii S: Molecular targeted therapies in hepatocellular carcinoma. Semin Oncol 39: 486-492, 2012.

8. Fry AM: The Nek2 protein kinase: A novel regulator of centrosome structure. Oncogene 21: 6184-6194, 2002.

9. Faragher AJ and Fry AM: Nek2A kinase stimulates centrosome disjunction and is required for formation of bipolar mitotic spindles. Mol Biol Cell 14: 2876-2889, 2003.

10. Bahe S, Stierhof YD, Wilkinson CJ, Leiss F and Nigg EA Rootletin forms centriole-associated filaments and functions in centrosome cohesion. J Cell Biol 171: 27-33, 2005.

11. Hayward DG, Clarke RB, Faragher AJ, Pillai MR, Hagan IM and Fry AM: The centrosomal kinase Nek2 displays elevated levels of protein expression in human breast cancer. Cancer Res 64: 7370-7376, 2004.

12. Wu G, Qiu XL, Zhou L, Zhu J, Chamberlin R, Lau J, Chen PL and Lee WH: Small molecule targeting the Hec1/Nek2 mitotic pathway suppresses tumor cell growth in culture and in animal. Cancer Res 68: 8393-8399, 2008.

13. Tsunoda N, Kokuryo T, Oda K, Senga T, Yokoyama Y, Nagino M, Nimura Y and Hamaguchi M: Nek2 as a novel molecular target for the treatment of breast carcinoma. Cancer Sci 100: 111-116, 2009.

14. Wang S, Li W, Lv S, Wang Y, Liu Z, Zhang J, Liu T and Niu Y: Abnormal expression of Nek2 and $\beta$-catenin in breast carcinoma: Clinicopathological correlations. Histopathology 59: 631-642, 2011.

15. de Vos S, Hofmann WK, Grogan TM, Krug U, Schrage M, Miller TP, Braun JG, Wachsman W, Koeffler HP and Said JW: Gene expression profile of serial samples of transformed B-cel lymphomas. Lab Invest 83: 271-285, 2003.

16. Neal CP, Fry AM, Moreman C, McGregor A, Garcea G, Berry DP and Manson MM: Overexpression of the Nek2 kinase in colorectal cancer correlates with beta-catenin relocalization and shortened cancer-specific survival. J Surg Oncol 110 828-838, 2014

17. Ning Z, Wang A, Liang J, Liu J, Zhou T, Yan Q and Wang Z: Abnormal expression of Nek2 in pancreatic ductal adenocarcinoma: A novel marker for prognosis. Int J Clin Exp Pathol 7: $2462-2469,2014$

18. Zhong X, Guan X, Dong Q, Yang S, Liu W and Zhang L: Examining Nek2 as a better proliferation marker in non-small cell lung cancer prognosis. Tumour Biol 35: 7155-7162, 2014.

19. Djebali S, Davis CA, Merkel A, Dobin A, Lassmann T, Mortazavi A, Tanzer A, Lagarde J, Lin W, Schlesinger F, et al: Landscape of transcription in human cells. Nature 489: 101-108, 2012.

20. Ozsolak F and Milos PM: RNA sequencing: Advances, challenges and opportunities. Nat Rev Genet 12: 87-98, 2011.

21. Sultan M, Schulz MH, Richard H, Magen A, Klingenhoff A, Scherf M, Seifert M, Borodina T, Soldatov A, Parkhomchuk D, et al: A global view of gene activity and alternative splicing by deep sequencing of the human transcriptome. Science 321 : 956-960, 2008
22. Ferreira PG, Jares P, Rico D, Gómez-López G, MartínezTrillos A, Villamor N, Ecker S, González-Pérez A, Knowles DG, Monlong J, et al: Transcriptome characterization by RNA sequencing identifies a major molecular and clinical subdivision in chronic lymphocytic leukemia. Genome Res 24: 212-226, 2014.

23. Rezaeian I, Tavakoli A, Cavallo-Medved D, Porter LA and Rueda L: A novel model used to detect differential splice junctions as biomarkers in prostate cancer from RNA-Seq data. J Biomed Inform 60: 422-430, 2016.

24. Huang Q, Lin B, Liu H, Ma X, Mo F, Yu W, Li L, Li H, Tian T, Wu D, et al: RNA-Seq analyses generate comprehensive transcriptomic landscape and reveal complex transcript patterns in hepatocellular carcinoma. PLoS One 6: e26168, 2011.

25. Xavier LL, Viola GG, Ferraz AC, Da Cunha C, Deonizio JM, Netto CA and Achaval M: A simple and fast densitometric method for the analysis of tyrosine hydroxylase immunoreactivity in the substantia nigra pars compacta and in the ventral tegmental area. Brain Res Brain Res Protoc 16: 58-64, 2005.

26. Das TK, Dana D, Paroly SS, Perumal SK, Singh S, Jhun H, Pendse J, Cagan RL, Talele TT and Kumar S: Centrosomal kinase Nek2 cooperates with oncogenic pathways to promote metastasis. Oncogenesis 2: e69, 2013.

27. Zhou W, Yang Y, Xia J, Wang H, Salama ME, Xiong W, Xu H, Shetty S, Chen T, Zeng Z, et al: NEK2 induces drug resistance mainly through activation of efflux drug pumps and is associated with poor prognosis in myeloma and other cancers. Cancer Cell 23: 48-62, 2013.

28. Chien CS, Shen KH, Huang JS, Ko SC and Shih YW: Antimetastatic potential of fisetin involves inactivation of the PI3K/Akt and JNK signaling pathways with downregulation of MMP-2/9 expressions in prostate cancer PC-3 cells. Mol Cell Biochem 333: 169-180, 2010.

29. O'Connell MJ, Krien MJ and Hunter T: Never say never. The NIMA-related protein kinases in mitotic control. Trends Cell Biol 13: 221-228, 2003.

30. Liu Q, Hirohashi Y, Du X, Greene MI and Wang Q: Nek2 targets the mitotic checkpoint proteins Mad2 and Cdc20: A mechanism for aneuploidy in cancer. Exp Mol Pathol 88: 225-233, 2010.

31. Gräf R: DdNek2, the first non-vertebrate homologue of human Nek2, is involved in the formation of microtubule-organizing centers. J Cell Sci 115: 1919-1929, 2002.

32. Prigent C, Glover DM and Giet R: Drosophila Nek2 protein kinase knockdown leads to centrosome maturation defects while overexpression causes centrosome fragmentation and cytokinesis failure. Exp Cell Res 303: 1-13, 2005.

33. Liu X, Gao Y, Lu Y, Zhang J, Li L and Yin F: Upregulation of NEK2 is associated with drug resistance in ovarian cancer. Oncol Rep 31: 745-754, 2014

34. Naro C, Barbagallo F, Chieffi P, Bourgeois CF, Paronetto MP and Sette C: The centrosomal kinase NEK2 is a novel splicing factor kinase involved in cell survival. Nucleic Acids Res 42: 3218-3227, 2014.

35. Takahashi Y, Iwaya T, Sawada G, Kurashige J, Matsumura T, Uchi R, Ueo H, Takano Y, Eguchi H, Sudo T, et al: Up-regulation of NEK2 by microRNA-128 methylation is associated with poor prognosis in colorectal cancer. Ann Surg Oncol 21: 205-212, 2014.

36. Marina M and Saavedra HI: Nek2 and Plk4: Prognostic markers, drivers of breast tumorigenesis and drug resistance. Front Biosci (Landmark Ed) 19: 352-365, 2014

37. Gao J, Ding F, Liu Q and Yao Y: Knockdown of MACC1 expression suppressed hepatocellular carcinoma cell migration and invasion and inhibited expression of MMP2 and MMP9. Mol Cell Biochem 376: 21-32, 2013.

38. Li J, Lau GK, Chen L, Dong SS, Lan HY, Huang XR, Li Y, Luk JM, Yuan YF and Guan XY: Interleukin 17A promotes hepatocellular carcinoma metastasis via NF- $\kappa \mathrm{B}$ induced matrix metalloproteinases 2 and 9 expression. PLoS One 6: e21816, 2011. 